\title{
Marie-Françoise Guédon
}

University of British Columbia (1982)

Anthropologue, professeure agrégée, Département d'études anciennes et de sciences des religions, Université d'Ottawa (2008)

$(1982)$

\section{"Problèmes de définition du chamanisme chez les Amérindiens de la côte Nord-Ouest, l'exemple des Tsimshian."}

\author{
Un document produit en version numérique par Jean-Marie Tremblay, bénévole, \\ professeur de sociologie au Cégep de Chicoutimi \\ Courriel: jean-marie_tremblay@uqac.ca \\ Site web pédagogique : http://www.uqac.ca/jmt-sociologue/ \\ Dans le cadre de: "Les classiques des sciences sociales" \\ Une bibliothèque numérique fondée et dirigée par Jean-Marie Tremblay, \\ professeur de sociologie au Cégep de Chicoutimi \\ Site web: http://classiques.uqac.ca/ \\ Une collection développée en collaboration avec la Bibliothèque \\ Paul-Émile-Boulet de l'Université du Québec à Chicoutimi \\ Site web: http://bibliotheque.uqac.ca/
}




\section{Politique d'utilisation de la bibliothèque des Classiques}

Toute reproduction et rediffusion de nos fichiers est interdite, même avec la mention de leur provenance, sans l'autorisation formelle, écrite, du fondateur des Classiques des sciences sociales, Jean-Marie Tremblay, sociologue.

Les fichiers des Classiques des sciences sociales ne peuvent sans autorisation formelle:

- être hébergés (en fichier ou page web, en totalité ou en partie) sur un serveur autre que celui des Classiques.

- servir de base de travail à un autre fichier modifié ensuite par tout autre moyen (couleur, police, mise en page, extraits, support, etc...),

Les fichiers (.html, .doc, .pdf., .rtf, .jpg, .gif) disponibles sur le site Les Classiques des sciences sociales sont la propriété des Classiques des sciences sociales, un organisme à but non lucratif composé exclusivement de bénévoles.

Ils sont disponibles pour une utilisation intellectuelle et personnelle et, en aucun cas, commerciale. Toute utilisation à des fins commerciales des fichiers sur ce site est strictement interdite et toute rediffusion est également strictement interdite.

L'accès à notre travail est libre et gratuit à tous les utilisateurs. C'est notre mission.

Jean-Marie Tremblay, sociologue

Fondateur et Président-directeur général, LES CLASSIQUES DES SCIENCES SOCIALES. 
Cette édition électronique a été réalisée par Jean-Marie Tremblay, bénévole, professeur de sociologie au Cégep de Chicoutimi à partir de :

Marie-Françoise Guédon

[Anthropologue, professeure agrégée, Département d'études anciennes et de sciences des religions, Université d'Ottawa]

"Problèmes de définition du chamanisme chez les Amérindiens de la côte Nord-Ouest, l'exemple des Tsimshian.”

Un article publié dans la revue CULTURE, Numéro spécial, vol. II, no 3, 1982, “Nations amérindiennes au Canada”, pp. 129-143. Société canadienne d'ethnologie.

[Autorisation formelle accordée par l'auteur le 26 juillet 2008 de diffuser cet article dans Les Classiques des sciences sociales.]

Courriel : mguedon@uOttawa.ca

Polices de caractères utilisée :

Pour le texte: Times New Roman, 12 points.

Pour les citations : Times New Roman, 12 points.

Pour les notes de bas de page : Times New Roman, 12 points.

Édition électronique réalisée avec le traitement de textes Microsoft Word 2004 pour Macintosh.

Mise en page sur papier format : LETTRE (US letter), 8.5’’ x 11'’)

Édition numérique réalisée le 3 août 2008 à Chicoutimi, Ville de Saguenay, province de Québec, Canada.

\section{Fait avec}

Macintosh 


\section{Marie-Françoise Guédon}

University of British Columbia (1982)

Anthropologue, professeure agrégée, Département d'études anciennes et de sciences des religions, Université d'Ottawa (2008)

\section{"Problèmes de définition du chamanisme chez les Amérindiens de la côte Nord-Ouest, l'exemple des Tsimshian”}

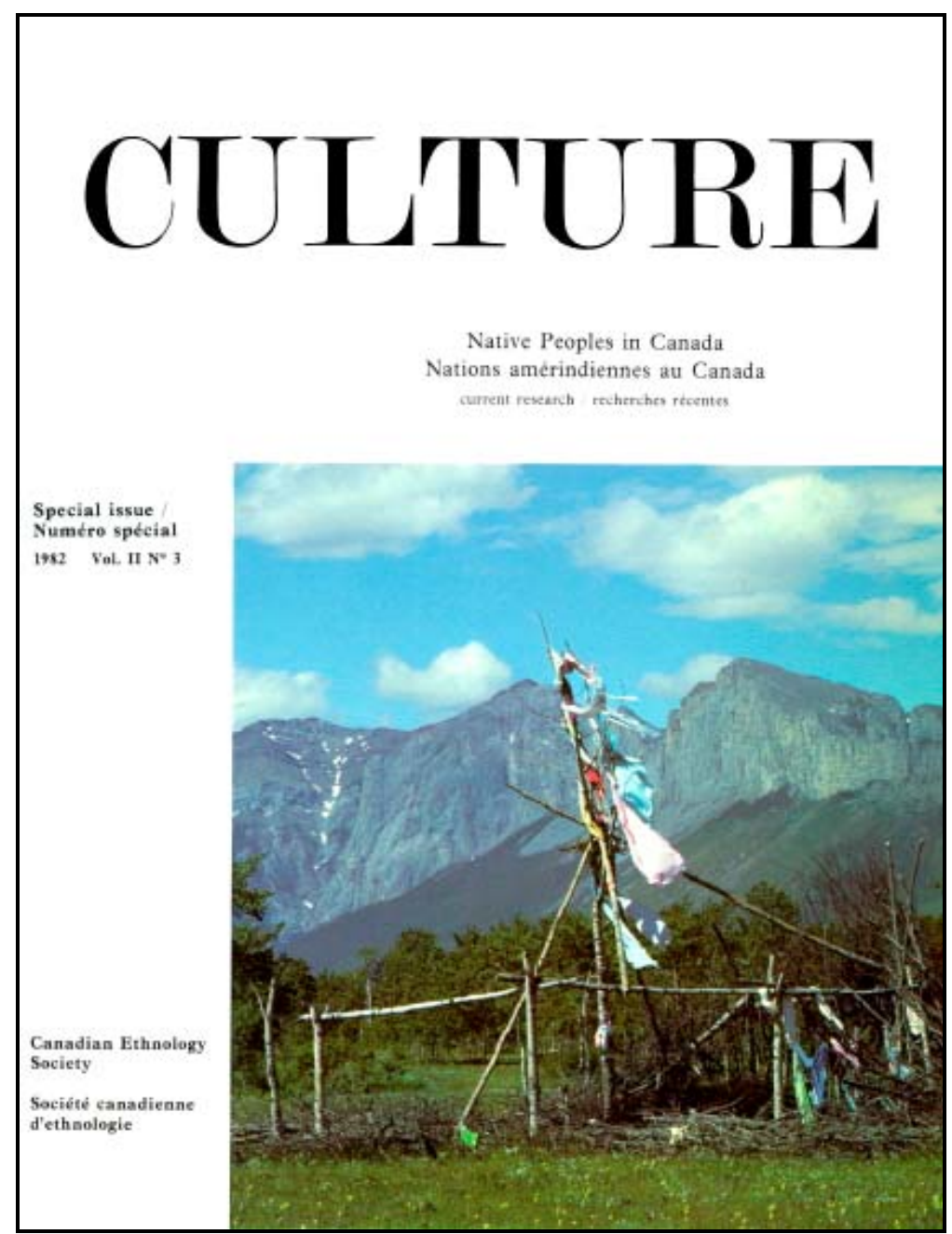

Un article publié dans la revue CULTURE, Numéro spécial, vol. II, no 3, 1982, "Nations amérindiennes au Canada”, pp. 129-143. Société canadienne d'ethnologie. 


\section{Table des matières}

Résumé

Tableau I. résumé des caractéristiques générales qui se retrouvent d'une culture à l'autre, y compris les Tsimshian, et qui distinguent le chamane des autres membres de sa communauté.

Tableau II. Résumé de la situation au moment de l'expansion maximale des sociétés secrètes qui eut lieu au moment de l'arrivée des commerçants euro-américains.

$\underline{\text { Références }}$ 


\section{Marie-Françoise Guédon \\ University of British Columbia (1982) \\ Anthropologue, professeure agrégée, Département d'études anciennes et de sciences des religions, Université d'Ottawa (2008) \\ "Problèmes de définition du chamanisme chez les Amérindiens de la côte Nord-Ouest, l'exemple des Tsimshian”.}

Un article publié dans Nouvelles technologies et société. Actes du colloque du 45e anniversaire de fondation de la Faculté des sciences sociales de l'Université Laval, pp 28-30. Québec: Faculté des sciences sociales, Université Laval, 1985, $306 \mathrm{pp}$.

L'auteur expose ici un problème complexe, celui de la définition du chamanisme. Ce mot, d'origine toungouse, s'applique aujourd'hui indifféremment à plusieurs catégories religieuses. Prenant exemple sur les Tsimshian de la côte Nord-Ouest, l'auteur montre comment ce problème complexe doit être analysé de manière ponctuelle et structurée.

This papier deals with a complex issue, that of the definition of shamanism. This term, of Toungous origin, is generally applied today to a variety of religious categories. Taking as an example the Tshimshian of the Northwest coast, the author shows to what extent this complex problem must be analyzed in a precise and structured way.

Parler de chamanisme en Amérique du Nord ouvre la voie à toutes sortes de discussions puisque, ce faisant, on étend à tout un continent le sens d'un terme qui s'appliquait au départ aux guérisseurs toungouses de Sibérie centrale. L'usage établi aujourd'hui nous permet de parler de chamanisme australien comme de chamanisme japonais ; il peut nous faire oublier que le chamanisme ne correspond pas à une cosmologie ou doctrine précise et qu'il existe en fait autant de chamanismes que de cultures supportant la présence de chamanes. On a pu présenter le chamanisme, entre autres, comme une religion, sinon la religion primitive, comme 
une philosophie plus ou moins archaïque ${ }^{1}$, comme une maladie similaire à l'épilepsie ou à la schizophrénie ou comme une série de traditions supportant les activités de guérisseurs ${ }^{2}$. je le définirai plus étroitement comme un système de croyances, dont celles relatives à l'existence indépendante d'une âme humaine séparable du corps, de méthodes et techniques mentales et rituelles ayant pour but le développement et le contrôle de facultés et pouvoirs surhumains incluant toujours le don de guérir les malades; il fait habituellement partie d'un contexte culturel dépendant d'une économie de chasse, pêche et cueillette et insiste sur les pouvoirs obtenus par l'individu qui a réussi à entrer en contact personnel avec le monde non-humain. Un tel système fait partie des cultures amérindiennes de la côte du Pacifique du Canada et de l'Alaska, c'est-à-dire la côte Nord-Ouest. Mais toute étude chamanique dans cette région se révèle à la fois fascinante et frustrante du point de vue de l'auteur comme de celui du lecteur, et cela pour un éventail de raisons qui ont influencé et influencent encore les recherches dans ce domaine et les domaines voisins.

En ce qui concerne les Amérindiens de la côte Nord-Ouest, un examen de la littérature anthropologique met immédiatement le lecteur en contact avec une réalité culturelle extrêmement riche. L'arrivée des colons européens a été tardive mais brutale, et son effet dévastateur. Une grande partie de ces cultures a disparu. Les recherches ethnographiques sont gênées par la christianisation qui a eu souvent pour corollaire une redéfinition des pratiques cérémonielles et des croyances qui sont devenues pour nombre d'autochtones des superstitions païennes dont beaucoup d'informateurs préfèrent ne pas parler. En dépit de cela, la richesse, la flamboyance et la diversité de la vie cérémonielle - depuis les traditions qui n'existent plus que dans la mémoire d'une poignée d'individus jusqu'aux rituels

1 Mircea Eliade, 1951. Voir aussi entre autres The way of the Shaman de Michael Harner, 1980 ; Shamanic Voices of Joan Halifax, 1979 ; et The Shaman's Doorway de Stephen Larsen, 1976.

2 Voir par exemple Eliade : Recent Works on Shamanism : A Review Article, 1961 ; et Czaplicka : Aboriginal Siberia, 1914. Ohlmarks a basé sa distinction de chamanismes arctique et subarctique sur le degré d'hystérie provoqué par l'environnement naturel ; pour des recherches plus sérieuses et plus récentes, voir Stanislav Groff : Realms of the human unconscious, observations from LSD research, 1976. 
inventés sur place aujourd'hui par les participants des nouvelles cérémonies -, restent évidentes.

En dehors des grandes monographies classiques comme celles de Franz Boas, John Swanton, Phillip Drucker, Viola Garfield, Thomas Mclllwraith ou Frederica de Laguna, la plupart des études plus récentes se concentrent sur les problèmes de définition et d'organisation des groupes de parenté, des classes ou rangs sociaux, des systèmes de chefferie, ou bien sur la présentation de cérémonies qui font partie des cycles des fêtes regroupés sous le terme de potlatch, ou bien encore et surtout sur l'art - en particulier les masques - et ses relations avec la cosmologie, la technologie ou l'organisation sociale. Le chamanisme n'est pas oublié mais il est habituellement pris pour acquis, et rares sont les ethnologues ou même les ethnographes - classiques ou modernes - qui sentent le besoin de le définir ou de le décrire. Cela bien que les ethnographies les plus précises et les plus détaillées aient démontré la nécessité de respecter le caractère émique des données chamaniques et aient révélé l'ambiguïté des catégories générales, y compris celles de « chamane » et de « chamanisme ». La plupart des descriptions sont trop générales, coincées, d'une part, entre l'examen détaillé du côté plus spectaculaire de la vie cérémonielle qu'offrent les potlatches et les activités des sociétés de danseurs ou sociétés secrètes et, d'autre part, entre les présentations de la cosmologie ou des cycles de mythes. Paradoxalement, le terme chamane est utilisé de façon si fréquente que le concept de chamanisme s'estompe et devient de plus en plus flou. La multiplicité des activités rituelles forme un obstacle important à la clarification de la situation. En particulier, les sociétés secrètes sont tantôt incluses, tantôt exclues des manifestations du chamanisme dans la région. Une définition adéquate du chamanisme demande donc l'examen de tout un contexte cérémoniel, contexte qui varie d'ailleurs de culture en culture. Il nous faut noter des exceptions à ce manque de précisions : le compte rendu de Franz Boas et George Hunt sur la religion kwakiutl comprend toute une section sur le chamanisme décrit du point de vue d'un apprenti-guérisseur (George Hunt lui-même ${ }^{3}$ ); Marius Barbeau a publié l'autobiographie d'un chamane tsimshian ${ }^{4}$; enfin Frederica de Laguna, dans une monographie monumentale en trois volumes (si précise qu'elle nous donne le dé-

3 Franz Boas et George Hunt : Religion of the Kwakiutl Indians, 1930.

4 Marius Barbeau : Medicine Men of the North Pacific Coast, 1958. 
tail de notes de terrain et des déclarations des informateurs), réussit à nous donner une image satisfaisante des chamanes chez les Tlingit Yakutat ${ }^{5}$. Depuis peu, la situation s'est améliorée, de plus en plus d'ethnologues et d'étudiants s'intéressent au chamanisme de la côte Nord-Ouest. Tout d'abord, les ethnologues d'aujourd'hui, qui sont en contact avec les communautés indiennes, tiennent compte de la survie d'activités rituelles traditionnelles réinterprétées par les participants dans un contexte moderne. Tandis que certains décrivent les manifestations modernes de ces activités (cf Michael et Della Kew) ou tentent d'expliquer cette continuité (cf Wayne Suttles chez les Salish et Pamela Amoss chez les Nooksak), d'autres veulent reconstruire ces systèmes traditionnels de pensée (cf Irving Goldman ou Martin Reid chez les Kwakiutl), ou essaient de percer le mystère qui entoure des cosmogonies extrêmement fertiles sur le plan philosophique comme sur le plan rituel et artistique (cf Wilson Duff). Plus d'une vingtaine de thèses de maîtrise ou de doctorat ont été écrites durant les dix dernières années sur le chamanisme ou des sujets connexes. Les travaux de Victor Turner sur le concept de liminalité, de Mary Douglas sur les rituels et les symboles, d'Erika Bourguignon sur les états de transe et tout récemment de Kennelm Burridge sur le concept d'individu et de personne ainsi, bien sûr, que la pensée de Claude Lévi-Strauss, influencent une grande partie de ces thèses. Ensuite, les spécialistes de l'art amérindien ont redécouvert le sacré et un point de vue religieux dans les oeuvres des artistes de la côte Nord-Ouest. Comme l'explique Aldona Jonaitis dans un article sur les diverses façons dont on a interprété l'art amérindien, les facteurs qui sont intervenus pour créer une atmosphère dans. laquelle pouvait se développer cette nouvelle signification du chamanisme «sont tous reliés au sens qu'a le chamanisme pour l'homme blanc » ${ }^{6}$, et ce sont nos propres bouleversements sociaux et intellectuels que nous devons lire d'abord dans cette évolution (Jonaitis, 1981 : 58). je suis d'accord avec elle, car ce nouvel intérêt pour le chamanisme amérindien s'étend à bien d'autres domaines qu'à l'art. Il facilite les contacts entre chercheurs, mais pose de sérieux problèmes à l'ethnologue qui a besoin de données précises, car les définitions très générales qu'utilisent la plupart des auteurs, pour qui tout ce qui touche de près ou de loin au sacré devient « chamanique », n'ont qu'un lointain

5 Frederica de Laguna, Under the Shadow of Mount Saint Elias, 1972.

6 Aldona Jonaitis, Creations of Mystics and Philosophers : The White Man's Perceptions of North West Coast Indian Art from the 1930s to the present. 1982. 
rapport avec les descriptions que peuvent encore fournir les informateurs indiens. Un certain nombre d'ethnologues, dont je suis, préféreraient redéfinir le chamanisme nord-américain d'après les concepts élaborés par leurs informateurs et reconsidérer les données ethnographiques avant de parler du chamanisme comme d'un phénomène universel et surtout homogène.

La côte Nord-Ouest du Pacifique offre à cet égard un terrain de recherche privilégié. Si on la compare au reste du Canada, cette aire culturelle se caractérise en effet par un morcellement linguistique et culturel extrême. Au moins trente dialectes, répartis en six grandes familles linguistiques, sont parlés encore aujourd'hui par les Indiens de la Colombie britannique seulement : Wakashan (Nootka, Kwakiutl du nord et du sud), Salish (Salish de l'intérieur, Salish côtiers, Bella Coola) et les trois groupes du nord, Tsimshian, Haida et Tlingit, auxquels s'ajoutent les Kutenai au sud-est et bien sûr les Athapaskan des plateaux de l'intérieur. Cette diversité correspond-elle à des variations culturelles ? Plus précisément, pouvonsnous parler d'un chamanisme en général ou de chamanismes particuliers ? Les contacts entre communautés indigènes à l'époque préhistorique ou historique qu'il s'agisse de commerce, de guerre, de diplomatie, de mariage ou d'échanges cérémoniels -ont certainement contribué à la dissémination de nombreuses pratiques et de rituels, mais l'habitude qu'ont prise de nombreux ethnologues de présenter la région tout entière comme une seule entité culturelle plus ou moins cohérente, et le manque d'informations - endémique dans cette région et ce domaine nous empêchent d'évaluer l'importance de ces échanges ainsi que la façon souvent très formelle qui présidait pour les autochtones à tout emprunt d'élément cérémoniel. Mes Propres recherches sur le terrain m'ont convaincue de la nécessité de respecter à la fois les particularismes locaux (et l'autonomie culturelle de chaque communauté) et le jeu quasi historique des échanges de rituels, pratiques, chants, danses, textes, et autres privilèges cérémoniaux, échanges qui compliquent l'évolution locale des institutions y compris celles liées au chamanisme.

Les populations de la côte et des grandes vallées sont presque sédentaires, la majorité vivant la plus grande partie de l'année et surtout l'hiver dans des villages dont l'emplacement est fixe ; les territoires de pêche, de chasse et de cueillette sont strictement délimités par des frontières définies par des accords entérinés par des traités dont le souvenir est perpétué de cérémonies en cérémonies, un aspect 
essentiel des grandes fêtes appelées communément potlatch. Correspondant à ces divisions territoriales, un système social complexe, comprenant des groupes de parenté dont la composition varie de peuple en peuple mais qui reste souvent unilinéaire, structure et hiérarchise les relations sociales, définit la position de chaque individu à l'intérieur des séries de rangs, de titres et de privilèges, qui composent une partie du patrimoine héréditaire de chaque groupe de parenté, et confirme les droits territoriaux de chacun. Il encadre aussi les relations entre communautés voisines et même celles de langues et cultures différentes ; ces relations sont régies par des droits et privilèges diplomatiques, commerciaux et cérémoniels détenus par les chefs et familles nobles. Ce système est très ancien : les recherches archéologiques ont montré que nous sommes en présence de cultures relativement stables établies depuis au moins deux millénaires. Mais en dépit de leurs différences, elles sont dépendantes les unes des autres. Des chocs culturels comme ceux qui ébranlèrent la Californie au moment de l'arrivée des Espagnols, ou les Aléoutiennes alors que les activités des marchands russes commençaient à se faire sentir en Sibérie orientale ${ }^{7}$, ont été perçus par chacun des peuples de la côte NordOuest. L'équilibre commercial, guerrier et cérémoniel est toujours à refaire, mais tout changement s'inscrit rapidement dans les droits territoriaux d'une part et les privilèges rituels d'autre part, et se retrouve mentionné directement ou indirectement dans les mythes relatant l'origine et l'histoire de chacun des groupes de parenté. Dans ce contexte, si différent du reste du Canada, les traditions orales sont assez précises et assez tenaces pour donner à ces cultures une vue presque historique de leurs sociétés. L'histoire culturelle de la côte du Nord-Ouest est non seulement longue et détaillée, elle fait explicitement partie des traditions des cultures qui nous concernent, et son étude est essentielle pour comprendre la vie cérémonielle de ces peuples, y compris les activités chamaniques. Cette histoire est aussi difficile d'accès car, en tant que tradition orale, une grande partie des informations sont insérées dans les textes, chants, danses et autres privilèges qui, tant que la

7 Ces thèmes ont été développés par l'archéologue George MacDonald, du Musée national de l'Homme à Ottawa, qui travaille en ce moment sur l'évolution des cultures tsimshian durant les deux derniers millénaires et les cinq cents dernières années en particulier, et qui s'intéresse entre autres aux répercussions qu'ont eues les mouvements économiques, démographiques et politiques européens sur les peuples des côtes du Pacifique. 
tradition est vivante, restent la propriété des lignages ou des familles qui en contrôlent l'accès.

Les Tsimshian offrent une illustration précise de la complexité de la société dans laquelle opère le chamane indien de la côte Nord-Ouest. Les Tsimshian tiennent parmi les peuples du Nord une position centrale ; situés le long de la côte entre les Kwakiutl du nord (Bella Bella, Haisla, Heiltsuk, voisins des Bella Coola) et les Tlingit de l'Alaska, en face des Haida des îles de la Reine-Charlotte, ils occupent aussi les vallées de la rivière Nass et de la rivière Skeena qui conduisent aux territoires athapaskan des Porteurs et des Sékanis. Parmi les ethnologues qui ont travaillé dans cette région, Franz Boas, Viola Garfield, et Marius Barbeau, assisté de William Beynon, ont été les principaux auteurs, et probablement les seuls, qui ont eu l'occasion de décrire des séances chamaniques qui avaient encore lieu au moment où ils écrivaient. (Ce qui ne veut pas dire que l'information est absente aujourd'hui, mais plutôt qu'elle est plus difficile à obtenir.) Leurs écrits forment aujourd'hui encore la base des données dont nous disposons. Depuis 1970, les recherches ont repris un nouvel essor, grâce aux initiatives locales, et à la curiosité des anthropologues. Ainsi une dizaine d'ethnologues et de linguistes ont travaillé avec l'un ou l'autre des groupes, les archéologues ont procédé aux fouilles, permettant à notre connaissance des Tsimshian de s'accroître peu à peu.

Les trois principaux dialectes tshimshian correspondent aux trois peuples des Gitksan (le long de la rivière Skeena), des Niska (à l'embouchure de la rivière Nass) et des Tsimshian de la côte. Chaque peuple est divisé en quatre phratries matrilinéaires exogamiques qui se correspondent dans les trois cas, et correspondent aussi aux moitiés des Haida et des Tlingit avec lesquels les Tsimshian sont liés par des relations commerciales, des alliances matrimoniales et, dans le passé, des attaques plus ou moins fréquentes, auxquelles s'ajoutent l'histoire commune d'un certain nombre de lignages originaires du Nord, ou ayant voyagé d'un pays à l'autre. Les phratries (identifiées aujourd'hui par les grands blasons du Loup, de la Grenouille ou du Corbeau, de l'Aigle, et de l'Orque ou de l'Épilobe) regroupent des lignages matrilinéaires localisés (parfois groupés en clans) que les Tsimshian appellent wilp ou Maison. Chaque village comprend au moins trois de ces Maisons. Chaque lignage ou Maison possède une série de noms ou titres correspondant à des territoires donnés, y compris des droits de pêche et chasse, et à des bla- 
sons précis. Les chefs de lignage sont en général les plus hautes autorités ; ils acquièrent leurs rôles et fonctions au moment où ils accèdent aux titres les plus élevés du lignage. Mais chez les Tsimshian de la côte, certains chefs de lignage ont réussi dans le passé à faire reconnaître leur autorité par d'autres lignages et même des lignages appartenant à d'autres phratries, prenant en même temps le contrôle de certaines fonctions religieuses au niveau du village ou même de groupes de villages.

Au développement des groupes de parenté, de la hiérarchie sociale et des séries de titres et blasons, correspond en effet chez les Tsimshian, comme tous les peuples de la côte Nord-Ouest, une mainmise des chefs et personnes de haut rang sur les ressources du surnaturel. Il s'est donc constitué autour ou à côté des chamanes une série d'institutions qui ont repris, soit au compte des lignages ou des familles nobles, soit au compte de sociétés secrètes, les rituels de quête d'une vision ou d'un esprit-gardien, les rites de passages et autres cérémonies d'initiation, ainsi que les festivals ou Danses d'hiver. L'identité des gardiens surnaturels est maintenant souvent dictée au départ par les groupes de parenté ou même par le titre dont on peut espérer hériter à l'intérieur de ces groupes. Les sociétés secrètes, dont les rituels ont lieu en hiver, durant la saison sacrée, se définissent de façon théorique par opposition à l'organisation sociale séculaire ou profane des groupes de parentés et des villages, mais restent partout dominées par l'aristocratie, et renforcent la hiérarchie sociale bien plus qu'elles ne la contrarient. Elles ont conservé des liens étroits mais ambigus avec les chamanes dont elles utilisent d'ailleurs les pouvoirs quand elles ne les parodient pas. Les personnes investies du pouvoir de contrôler les quêtes de vision, de faire passer les jeunes à l'état adulte, ou d'initier les novices aux mystères des sociétés secrètes, tout comme celles, qui peuvent se prévaloir d'une rencontre fortuite ou non avec le surnaturel, jouissent d'un prestige certain, et elles sont - dans pratiquement toutes les langues de la côte - considérées comme appartenant à des catégories voisines. Un chef n'est pas un chamane mais il (ou elle) officie en qualité d'intermédiaire entre les humains et le nonhumain, et on l'appelle par un titre spécial qui comporte souvent le même terme que celui qui désigne les guérisseurs. je ne suis pas d'accord cependant avec Irving Goldman pour établir l'équation générale chef-charmane 8 : au contraire, je

8 Goldman remarque : 
pense que c'est seulement par l'étude des cosmologies propres à chaque culture de la région et à chacun des complexes rituels qui encadrent les définitions du chef, de l'initié, du chamane et des autres « techniciens du sacré » (pour reprendre l'expression d'Éliade) que l'on parviendra à mieux comprendre le chamanisme.

En langue tsimshian, le guérisseur homme ou femme, est appelé halaidm swanaskw, c'est-à-dire le « halait-qui-souffle ». Le terme halait s'applique à tous les personnages qui ont àfaire avec le sacré ou le non-humain et se traduit par « danse » ou «danseur » et fréquemment par le terme « chamane ». Parmi les termes qui dérivent de celui-ci, le terme simhalait (« le halait véritable ») désigne le chef qui officie dans la cérémonie du même nom. Cette cérémonie avait originellement pour but d'initier les enfants et débutants à la présence des puissances et esprits non-humains qu'ils (et elles) allaient rencontrer lors de rituels futurs. Le terme wihalait (« le grand halait») désigne les grands initiés et surtout les chefs initiateurs des sociétés secrètes. Tous ces «chamanes » ou «danseurs » sont engagés dans des contacts avec des entités qui sont désignées par le même terme, celui de naxnoq. Notons que les sorciers, qui empoisonnent leurs ennemis ou les rendent malades à l'aide de pratiques magiques basées sur l'utilisation de morceaux de cadavres, ne font pas partie des halait ; de plus la sorcellerie n'implique aucun contact avec les esprits ou êtres surnaturels. Le terme naxnok, traduit par Franz Boas et Viola Garfield par l'expression « être surnaturel » et par Marius Barbeau par le terme «esprit», peut aussi être compris comme voulant dire « pouvoir » ou « don », surtout un don sortant de l'ordinaire, et s'applique en général à des formes personnalisées des puissances non-humaines - esprits, animaux, êtres mythiques ou monstrueux et autres entités surhumaines. Ils se mani-

Among Kwakiutl the chiefs or the so-called "nobility", are equated with shamans. A chief is not necesarily a curing shaman and a curing shaman need not be a chief. In their major ritual roles, however, the chiefs are addressed as paxala (shaman) and behave as shamans. The supernatural powers of chiefs are akin to those of curing shamans, the "medicine men" (Goldman, 1975 : 4). Il conclut par cette équation :

Historically, the Kwakiutl shamanistic cult with its triple links to curing, chiefship, and life renewal belongs to the widespread shamanistic configuration reaching from eastern Siberia down to the American Southwest (Eliade 1964). Many tribes have extended the powers of the shaman from curing, divining, and weather control into broader reachers (Goldman, 1975 : 206). 
festent de temps à autre dans la perception d'un événement ou d'un être sortant de l'ordinaire ; ils peuvent aussi venir à l'aide des humains. En dépit du fait que le terme « chamane » a été utilisé par presque tous les ethnologues pour traduire des expressions qui, en langues amérindiennes, désignent les initiés, les ritualistes et même les chefs, je crois qu'une étude attentive de ces termes démontrerait qu'ils s'appliquent bien plus aux manifestations du surnaturel (ou du surhumain ou même du spirituel) en général qu'au monde chamanique en particulier.

En tout cas, le chamane guérisseur ne fait pas partie en tant que chamane du contexte social officiel, en particulier de la chefferie, des groupes de parenté et des sociétés secrètes ${ }^{9}$. Son statut social ${ }^{10}$, sa fonction cérémonielle et sa position dans le système cosmologique et philosophique de sa communauté, sont différents de ceux des autres ritualistes. Le tableau 1 résume les caractéristiques générales qui se retrouvent d'une culture à l'autre, y compris les Tsimshian, et qui distinguent, à mon avis, le chamane des autres membres de sa communauté.

Les rituels chamaniques confirment cette indépendance. Les séances de guérison, par exemple, ne sont contrôlées pour le contenu que par l'officiant lui-même et son client. Le scénario de l'action suit en général une progression classique : le diagnostic du problème se fait à l'aide de rêve, rêverie, ou vision ; il est suivi, soit de l'extraction de la maladie du corps du patient, soit de la recherche et, en cas de réussite, de la capture de l'âme censée être partie loin de son corps et du retour de l'âme fortifiée par le souffle du chamane. Ce scénario est appliqué de façon très libre et les techniques chamaniques varient considérablement d'un individu à l'autre. Cependant, en dépit des différents contextes culturels dans lesquels les chamanes opèrent, les techniques elles-mêmes se retrouvent d'une communauté à l'autre. À ce sujet, George MacDonald remarque que les chamanes communiquent

9 Ce que confirme par exemple le fait que le chamane ne tient pas compte des blasons et autres symboles des groupes de parenté dans le choix de ses assistants surnaturels et autres « charmes ».

10 Il est certain toutefois que la position de chamane pouvait donner lieu à certains avantages, ce qui a pu faire dire à Drucker qu'un individu, qui ne pouvait réussir socialement à cause d'une naissance trop basse, pouvait toujours essayer de se prévaloir de pouvoirs chamaniques et acquérir ainsi un certain prestige personnel. 
les uns avec les autres, échangeant techniques, trucs, connaissances médicales et rituels d'une culture à l'autre, et qu'ils jouissent d'une liberté peu commune dans leurs voyages. Il note aussi qu'à la différence d'une prêtrise ou d'une société secrète, le chamanisme insiste sur l'innovation dans ses techniques comme dans son fonctionnement (MacDonald, 1982 : 225). Une analyse des méthodes d'action du chamane lors des séances de guérison demanderait un appareil psychologique assez complexe. Seul Marius Barbeau, parmi les auteurs classiques, a reconnu la valeur de données telles le détail des visions, des processus mentaux et, en général, du monde intérieur du chamane. Mais l'accès au point de vue du chamane luimême, à son mode de relation avec son assistant, ou ses assistants surnaturels, nous amène à penser que les aspects les plus riches et peut-être les plus divers du chamanisme, ont été trop souvent négligés. Chez les chamanes tsimshian, l'assistant surnaturel, atiasxw, se révèle souvent être une image mentale ou représentation du problème ou de la maladie du patient plutôt qu'une entité indépendante du chamane et de sa situation. L'une des techniques les plus importantes utilisées par ces chamanes est la transposition du problème par le client du plan réel au plan mental et symbolique. Les images évoquées par le chamane pour exprimer sa perception de la situation sont ensuite résolues au moyen d'une action mimée dramatisée par l'officiant, ses assistants et même l'assemblée. Cette dramatisation peut même demander la participation des artistes qui travaillent pour les sociétés secrètes. Dans le contexte culturel des peuples de la côte et de leur immense richesse artistique (qu'il s'agisse de sculpture, de peinture, de danse, mime ou musique), cette technique de dramatisation a atteint un niveau de sophistication qu'on ne rencontre pas chez les Athapaskan du nord par exemple. (Son analyse demanderait la collecte de données que nous ne trouvons encore que rarement dans la littérature, quoiqu'elles soient encore présentes dans les cérémonies modernes.) C'est peut-être pour cette raison que la participation des chamanes au développement des arts est probablement plus prononcée ici qu'ailleurs dans le monde. Elle est déjà remarquablement présente dans les niveaux les plus anciens étudiés par les archéologues. 


\section{Tableau I}

Le tableau 1 résume les caractéristiques générales qui se retrouvent d'une culture à l'autre, y compris les Tsimshian, et qui distinguent, à mon avis, le chamane des autres membres de sa communauté.

$\underline{\text { Retour à la table des matières }}$

\begin{tabular}{|c|c|c|c|c|}
\hline & chamane & chef, noble & chef & initié \\
\hline fonction & guérisseur & $\begin{array}{l}\text { politique offi- } \\
\text { cielle }\end{array}$ & & $\begin{array}{l}\text { membre de la } \\
\text { société }\end{array}$ \\
\hline participants & $\begin{array}{l}\text { le malade et sa fa- } \\
\text { mille, maisonnée } \\
\text { ouverte au public }\end{array}$ & $\begin{array}{l}\text { nobles du ligna- } \\
\text { ge-hôte et des } \\
\text { lignages invités }\end{array}$ & $\begin{array}{l}\text { habitants du vil- } \\
\text { lage et leurs fa- } \\
\text { milles }\end{array}$ & $\begin{array}{l}\text { initiés du village } \\
\text { et novices }\end{array}$ \\
\hline but du rituel & $\begin{array}{l}\text { guérison du mala- } \\
\text { de, protection } \\
\text { contre sorciers et } \\
\text { autres forces mali- } \\
\text { gnes, contact avec } \\
\text { le surnaturel, les } \\
\text { animaux, les es- } \\
\text { prits, etc. }\end{array}$ & $\begin{array}{l}\text { spectacle, } \\
\text { confirmation } \\
\text { publique de pri- } \\
\text { vilèges héréditai- } \\
\text { res ou person- } \\
\text { nels }\end{array}$ & $\begin{array}{l}\text { initiation des } \\
\text { jeunes (rite de } \\
\text { passage) et leur } \\
\text { premier contact } \\
\text { officiel avec le } \\
\text { surnaturel pro- } \\
\text { tection morale, } \\
\text { propitiation du } \\
\text { gibier }\end{array}$ & $\begin{array}{l}\text { initiation de } \\
\text { nouveaux mem- } \\
\text { bres dans les } \\
\text { sociétés secrètes, } \\
\text { et acquisition de } \\
\text { nouveaux titres } \\
\text { pour les initiés }\end{array}$ \\
\hline $\begin{array}{l}\text { mode d'acqui- } \\
\text { sition du pou- } \\
\text { voir } \\
\text { l'officiant }\end{array}$ & $\begin{array}{l}\text { quête personnelle et } \\
\text { solitaire vision, } \\
\text { rêves et maladie }\end{array}$ & $\begin{array}{l}\text { héritage (lié au } \\
\text { titre) parfois } \\
\text { vision personnel- } \\
\text { le contrôlée par } \\
\text { lignage }\end{array}$ & héritage & $\begin{array}{l}\text { série d'initiations } \\
\text { successives } \\
\text { (avec enseigne- } \\
\text { ment formel) et } \\
\text { progression dans } \\
\text { la hiérarchie }\end{array}$ \\
\hline position & $\begin{array}{l}\text { nouveau nom utili- } \\
\text { sé lors des rituels } \\
\text { seulement (n'inter- } \\
\text { vient pas en tant } \\
\text { que chamane dans } \\
\text { les affaires du li- } \\
\text { gnage) }\end{array}$ & $\begin{array}{l}\text { nouveau nom } \\
\text { prérogatives } \\
\text { rang et respon- } \\
\text { sabilités }\end{array}$ & $\begin{array}{l}\text { proclamation du } \\
\text { titre de l'officiant } \\
\text { de ses privilèges } \\
\text { et responsabilités } \\
\text { morales }\end{array}$ & $\begin{array}{l}\text { nouveau titre à } \\
\text { l'intérieur de la } \\
\text { société }\end{array}$ \\
\hline candidats & $\begin{array}{l}\text { n'importe qui peut } \\
\text { être élu par le sur- } \\
\text { naturel y compris } \\
\text { un esclave }\end{array}$ & $\begin{array}{l}\text { nobles ou mem- } \\
\text { bres du lignage }\end{array}$ & $\begin{array}{l}\text { seuls les chefs de } \\
\text { très haut rang } \\
\text { peuvent officier } \\
\text { en tant que ritua- }\end{array}$ & $\begin{array}{l}\text { toute personne } \\
\text { assez riche pour } \\
\text { pouvoir payer } \\
\text { les dépenses des } \\
\text { cérémonies (en }\end{array}$ \\
\hline
\end{tabular}




\begin{tabular}{|c|c|c|c|c|}
\hline & chamane & chef, noble & chef & initié \\
\hline fonction & guérisseur & $\begin{array}{l}\text { politique offi- } \\
\text { cielle }\end{array}$ & & $\begin{array}{l}\text { membre de la } \\
\text { société }\end{array}$ \\
\hline \multirow{4}{*}{$\begin{array}{l}\text { accès à l'ini- } \\
\text { tiation }\end{array}$} & & \multirow{4}{*}{$\begin{array}{l}\text { pas d'initiation } \\
\text { véritable }\end{array}$} & \multirow[t]{3}{*}{ liste ou initiateur } & $\begin{array}{l}\text { pratique, les } \\
\text { nobles et enfants } \\
\text { de nobles) accè- } \\
\text { dent plus facile- } \\
\text { ment aux titres } \\
\text { les plus élevés) }\end{array}$ \\
\hline & $\begin{array}{l}\text { involontaire dans la } \\
\text { plupart des cas - } \\
\text { n'affecte que cer- } \\
\text { tains individus } \\
\text { (homme et femmes) }\end{array}$ & & & $\begin{array}{l}\text { volontaire sauf } \\
\text { en cas d'enlève- } \\
\text { ment par les } \\
\text { initiés }\end{array}$ \\
\hline & $\begin{array}{l}\text { sélection par les } \\
\text { puissances surnatu- } \\
\text { relles }\end{array}$ & & & \multirow{2}{*}{$\begin{array}{l}\text { hommes, surtout } \\
\text { pour les grades } \\
\text { élevés, femmes } \\
\text { admises dans les } \\
\text { grades moins } \\
\text { élevés initiation } \\
\text { rapide, publique, } \\
\text { avec torture, } \\
\text { privations, suivie } \\
\text { d'un enseigne- } \\
\text { ment formel (de } \\
\text { plusieurs semai- } \\
\text { nes) }\end{array}$} \\
\hline & $\begin{array}{l}\text { « initiation » per- } \\
\text { sonnelle, privée, } \\
\text { souvent fortuite } \\
\text { suivie d'un appren- } \\
\text { tissage de plusieurs } \\
\text { années }\end{array}$ & & $\begin{array}{l}\text { enseignement } \\
\text { formel pour les } \\
\text { novices (de plu- } \\
\text { sieurs jours) }\end{array}$ & \\
\hline $\begin{array}{l}\text { sources du } \\
\text { pouvoir }\end{array}$ & $\begin{array}{l}\text { « charme » ou « as- } \\
\text { sistant » : esprits } \\
\text { animaux de toutes } \\
\text { sortes, corps céles- } \\
\text { tes, phénomènes } \\
\text { naturels, objets et } \\
\text { outils dotés de pou- } \\
\text { voirs }\end{array}$ & $\begin{array}{l}\text { héritage confir- } \\
\text { mé par assem- } \\
\text { blée des nobles } \\
\text { et par « po- } \\
\text { tlatch » }\end{array}$ & $\begin{array}{l}\text { entité (ou initiés) } \\
\text { surnaturelle } \\
\text { apparaît sous } \\
\text { toutes sortes de } \\
\text { formes et de } \\
\text { normes puissan- } \\
\text { tes mais bénéfi- } \\
\text { ques }\end{array}$ & $\begin{array}{l}\text { montres mythi- } \\
\text { ques géants, } \\
\text { violents, destruc- } \\
\text { teurs, parce que } \\
\text { non-humains, } \\
\text { souvent canniba- } \\
\text { les, toujours } \\
\text { dangereux. }\end{array}$ \\
\hline $\begin{array}{l}\text { contact avec } \\
\text { le surnaturel }\end{array}$ & $\begin{array}{l}\text { contact direct avec } \\
\text { le monde non- } \\
\text { humain }\end{array}$ & $\begin{array}{l}\text { le contact n'est } \\
\text { que commémoré }\end{array}$ & $\begin{array}{l}\text { contact indirect } \\
\text { - par représenta- } \\
\text { tion ou dramati- } \\
\text { sation } \\
\text { - ou par l'inter- }\end{array}$ & $\begin{array}{l}\text { contact direct } \\
\text { puis indirect par } \\
\text { dramatisation } \\
\text { des pouvoirs } \\
\text { supposément }\end{array}$ \\
\hline
\end{tabular}




\begin{tabular}{|c|c|c|c|c|}
\hline & chamane & chef, noble & chef & initié \\
\hline \multirow[t]{2}{*}{ fonction } & guérisseur & $\begin{array}{l}\text { politique offi- } \\
\text { cielle }\end{array}$ & & $\begin{array}{l}\text { membre de la } \\
\text { société }\end{array}$ \\
\hline & & & $\begin{array}{l}\text { médiaire de l'of- } \\
\text { ficiant }\end{array}$ & reçus \\
\hline $\begin{array}{l}\text { état ou niveau } \\
\text { de conscience }\end{array}$ & $\begin{array}{l}\text { - transe légère et } \\
\text { contrôlée } \\
\text { - projection de la } \\
\text { conscience hors du } \\
\text { corps }\end{array}$ & $\begin{array}{l}\text { parfois transe } \\
\text { légère due à l'ef- } \\
\text { fet du masque et } \\
\text { du chant rythmé }\end{array}$ & $\begin{array}{l}\text { plein éveil (aussi } \\
\text { contrôlée que } \\
\text { possible) pour } \\
\text { l'officiant. Tran- } \\
\text { se (réelle ou } \\
\text { simulée) pour les } \\
\text { novices }\end{array}$ & $\begin{array}{l}\text { possession (réel- } \\
\text { le ou simulée), } \\
\text { frénésie ou exta- } \\
\text { se souvent vio- } \\
\text { lente contrôlée } \\
\text { de l'extérieur par } \\
\text { des assistants }\end{array}$ \\
\hline
\end{tabular}

L'ancienneté des activités chamaniques dans toute la région de la côte NordOuest n'est pas à démontrer ; les chamanes sont mentionnés dans pratiquement tous les mythes, y compris les grands mythes d'origine comme le cycle du corbeau - le corbeau étant d'ailleurs l'un des archétypes du chamane pour les peuples du Nord. Leur endurance face au processus de colonisation est moins connue. Pourtant, en dépit de l'opposition des autorités religieuses et administratives, des formes contemporaines des activités chamaniques adaptées à la situation démographique, sociale, économique et religieuse moderne, se sont développées dans beaucoup de cas, et cela dès l'arrivée des Européens. L'apport chrétien s'est immédiatement exprimé dans des syncrétismes qui ont laissé des traces dans les traditions actuelles, en particulier les activités des "prophètes » comme le fameux Bini qui opérait le long de la rivière Bulkley (Diamond Jenness, 1943). Il existe aussi des guérisseurs indiens chrétiens qui ne sont presque jamais mentionnés par les ethnologues alors qu'ils nous donnent un exemple remarquable de continuité culturelle. Il est vrai que dans bien des cas, et surtout dans les régions contrôlées par les églises protestantes, on se heurte à un tabou encore très puissant qui interdit aux informateurs chrétiens pratiquants de discuter de pratiques chamaniques. Quant aux formes traditionnelles, elles ont presque partout disparu sauf dans les plateaux de l'intérieur et là où les chamanes sont soutenus par la présence des sociétés secrètes. 
Les sociétés secrètes entretiennent avec les chamanes guérisseurs des relations ambiguës ; d'une part, la participation de chamanes guérisseurs aux cérémonies est nécessaire, et ces derniers sont souvent considérés comme membres de la société avant même leurs initiations bien qu'ils soient relégués à des niveaux inférieurs; d'autre part, les initiés se targuent de pouvoirs quasi-chamaniques du moins en apparence. L'essor, tardif, des sociétés secrètes n'aurait pu se faire sans l'expansion économique du XVIIIe siècle. Elles n'ont été détruites que lors de la désintégration de l'appareil social et politique qui supportait les grandes fêtes d'initiation ainsi que le « potlatch »et la transmission des droits et privilèges héréditaires, et elles font aujourd'hui une remontée spectaculaire dans les communautés du sud de la région : Nootka, Kwakiutl et surtout Salish. Chez les Kwakiutl, les rituels initiatiques renaissent, quoique de façon très abrégée, avec le développement du potlatch dont ils font maintenant partie et cela sous l'impulsion donnée par les artistes indiens aussi bien que par les chefs héréditaires. Chez les Salish enfin, on assiste depuis une dizaine d'années à une remontée rapide des rituels d'initiations ou «spirit dances »; cette renaissance est d'autant plus remarquable qu'elle s'accompagne de nouvelles interprétations, de l'intégration de divers rituels, y compris des rituels traditionnellement réservés aux guérisseurs, et d'une adaptation générale aux besoins et conditions de vie de la population autochtone actuelle ${ }^{11}$.

L'histoire des sociétés secrètes chez les Tsimshian est liée à celle des autres sociétés de la côte. Franz Boas a résumé, dans Religion of the Kwakiutl (1930) et dans The Social Organization and Secret Societies of the Kwakiutl Indians (1897), la répartition des sociétés secrètes dans les diverses communautés kwakiutl ainsi que chez leurs voisins ; Philip Drucker a détaillé, dans l'étude comparative Kwakiutl Dancing Societies, les éléments caractéristiques des sociétés secrètes de toute la côte pacifique canadienne. D'après Boas, comme d'après Drucker, la distribution géographique des sociétés secrètes reflètent leur évolution historique. On place habituellement leur origine chez les Kwakiutl du nord, c'est-à-dire les Bella Bella et les Heiltsuq qui occupent une position centrale entre les peuples du sud,

11 Wolfgang Jilek a mis en valeur l'aspect thérapeutique des «spirit dances» chez les Salish dans une étude récente (1982) qui examine les méthodes utilisées par les ritualistes salish pour contrecarrer les effets destructeurs de l'acculturation. 
ceux du nord, et les Bella Coola. Cette position a pu favoriser la floraison des rituels d'initiation qui semblent combiner toute une série de traditions différentes. En tout cas, ces rituels se retrouvent, sous une forme très atténuée, chez les Haida, et ils sont absents chez les Tlingit. Au sud, ils se développent de façon spectaculaire chez tous les peuples Kwakiutl, et chez quelques-uns des Salish et des Nootka où ils s'intègrent aux rites de passage et à la quête d'un esprit-gardien. Ils s'intègrent aussi aux rituels initiatiques Bella Coola à l'est et on en retrouve des traces chez les Athapaskan voisins des Tsimshian. En ce qui concerne les Tsimshian, immédiatement au nord des Bella Bella et Haisla, les sociétés secrètes se réduisent à deux sociétés principales auxquelles s'ajoutent un certain nombre de danses connexes, mais elles sont très actives et structurent une grande partie de la vie sociale et cérémonielle durant tout l'hiver.

Franz Boas 12 a résumé la distribution des sociétés secrètes en concluant qu'une comparaison des cérémonies chez tous ces peuples ne laisse aucun doute quant à leur origine commune. Les rituels sont semblables de l'Alaska au détroit de Juan de Fuca. Les danseurs portent partout des couronnes des bracelets, des colliers qui sont faits d'écorce de cèdre rouge teinte en rouge à l'aide de jus d'aulne. Partout des masques en bois sculpté et peints représentent les esprits. Des tambours en bois, des hochets (y compris des hochets sculptés en forme de crâne), et surtout des instruments à vent de toutes sortes construits sur le principe de la flûte (trompette, trompe, clarinette, et même hautbois), complètent l'essentiel de l'attirail nécessaire pour représenter les esprits. Mais l'indice le plus évident est

12 Boas écrit :

Among the Haida, Tlingit, and Tsimshian we find the names olala, me'iLa, and no'nLeM which belong to the ceremonial of the Kwakiutl as well. Among the Bilxula (Bella Coola) the names can not be derived from the same words as among the other tribes, but there the ceremonial itself is almost identical with that of the Kwakiutl. It certainly does not differ more from the ceremonial as described here than that of other tribes of Kwakiutl lineage differs from the ceremonial of the Kwaikiutl proper. Besides this, the names of the dancers, if not those of their dances, are very often borrowed from the Kwakiutl. Turning to the south, we find the Nootka as well as the Salishan tribes who practice the ceremonial, terming it by the two names Lo'koala and no'nLem, both of which are names used from portions of the ceremonial of the Kwakiutl (Boas, 1879 : 661). 
constitué par l'emploi de termes kwakiutl pour désigner les différentes sociétés, les grades d'initiés.

Cette progression vers le sud et le nord peut être vérifiée par l'étude des mythes d'origine des rituels d'initiation dans les diverses cultures en cause, et peut même être datée. D'après Franz Boas, la société des «Cannibales », qui est la plus prestigieuse des sociétés kwakiutl, est relativement récente et date des environs de 1835 pour les Kwakiutl, et 1825 pour les Tsimshian ${ }^{13}$. Chez les Bella Coola, les rituels des sociétés secrètes se retrouvent dans le complexe cérémonial du sisaok. McIlwraith a décrit le sisaok en détail, ajoutant qu'une grande partie de ce cérémonial aurait été importée des Bella Bella vers 1830 à la suite d'alliances matrimoniales ${ }^{14}$.

13 The Kwakiutl state uniformly that the custom of devouring men was introduced among their tribe about sixty years ago (ca 1835), and that is was derived from the Heiltsuq. We also have conclusive evidence that the custom was acquired by the Tsimshian not more than seventy years ago (ca. 1825) and that they also obtained it from the Heiltsuq (Boas, 1897 : 664).

14 The accounts of all informants agree that much of the sisaok ritual was newly imported into Bella Coola from Bella Bella According to some statements, Pottes, a Bella Coola chief who died between 1870 and 1880, married a Bella Bella wife, also one from Rivers Inlet, and it was these two women who brought with them as dower certain sisaok prerogatives. It is said that previous to that time the Bella Coola did not understand the use of the large cone-shaped whistles, nor were they able to summon the supernatural beings to the youths secluded in the back-roon; nor to dispel the beings at the conclusion of the rites (McIlwraith, 1948, II : 197). 


\section{Tableau II}

Résumé de la situation au moment de l'expansion maximale des sociétés secrètes qui eut lieu au moment de l'arrivée des commerçants euro-américains.

$\underline{\text { Retour à la table des matières }}$

Sociétés secrètes chez les Tsimshian

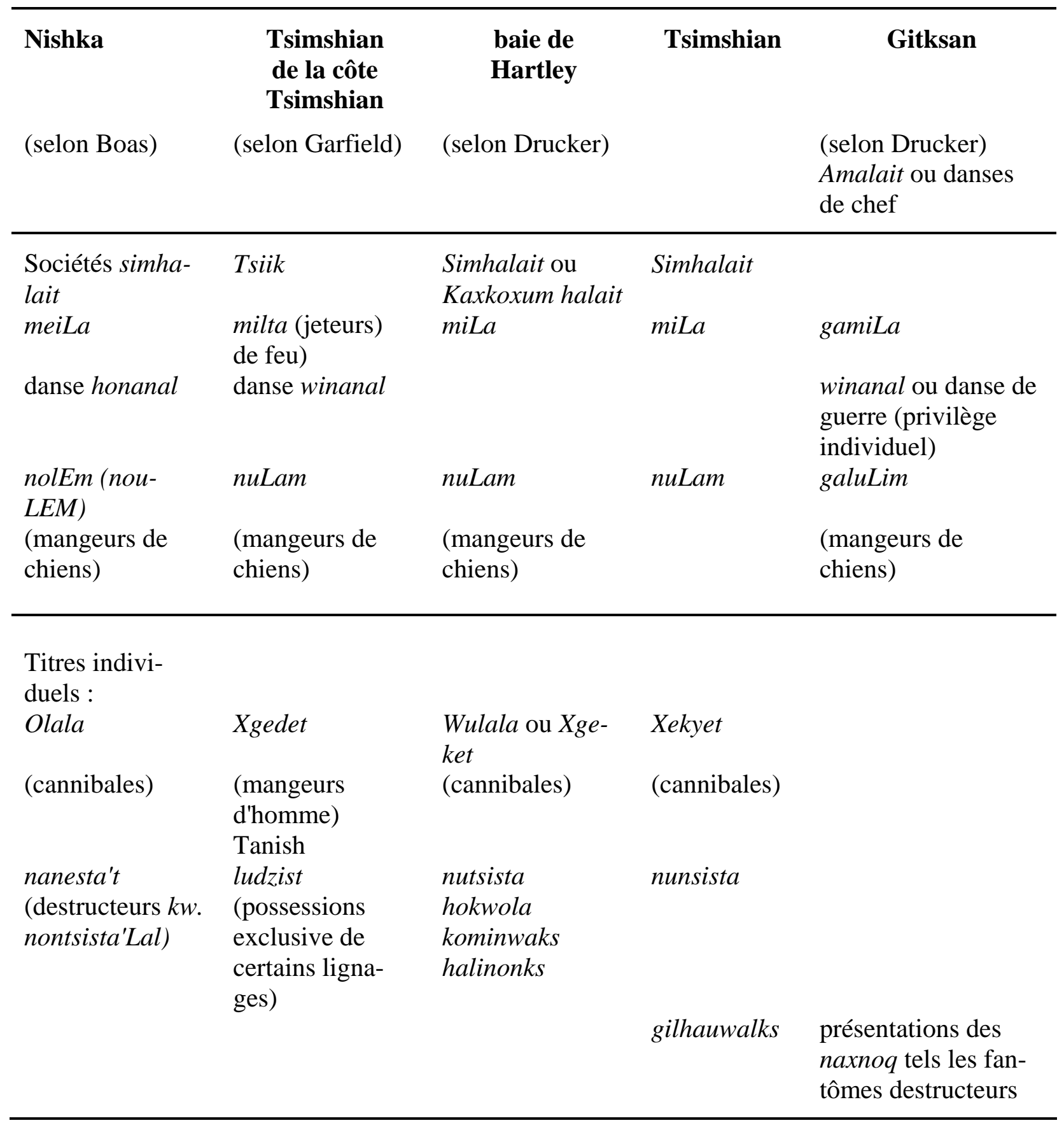


Toute description de ces cérémonies renvoie aux quêtes d'une vision ou d'un esprit, quêtes déjà pratiquées de façon individuelle par chaque adolescent et chaque adulte ambitieux. Viola Garfield 15 définit l'initiation dans les sociétés secrètes comme la quête formelle, planifiée et publique d'un esprit gardien. Le scénario de l'action est complexe ; il débute avec l'enlèvement des novices par les entités surnaturelles correspondant à la société dans laquelle ils vont être initiés. Les novices sont alors pris en charge par les ritualistes jusqu'à ce que l'esprit ou l'entité se manifeste en eux ; ils réapparaissent après plusieurs semaines dans un état de sauvagerie plus ou moins violente (mort symbolique). Ils sont ramenés progressivement à l'état humain, et interprètent au cours de danses les connaissances qu'ils viennent d'acquérir. Chez les Tsimshian, les sociétés secrètes se présentent de diverses façons selon qu'on les aborde dans un village ou un autre. Le tableau Il résume la situation au moment de l'expansion maximale des sociétés secrètes qui eut lieu au moment de l'arrivée des commerçants euro-américains.

Une série de mythes racontent les aventures des chefs tsimshian qui découvrirent les êtres surnaturels lesquels leur enseignèrent les danses des sociétés secrètes. De ces «danses »dérivent soit des sociétés comme les Nutlam (mangeurs de chiens) et les Mitla (danseurs), soit des danses qui font partie des prérogatives héréditaires associées au titre de certaines chefs ou contrôlées par certains ligna-

15 Novices were coached in every step of the initiation, from the preliminary bathing, fasting and purification to the final removal of spirit influence. The initiation was essentially a planned and formalized guardian spirit quest which was carried out in a public gathering rather than in solitude. The appropriate president spirit was called by the songs and symbolized by the dances of the members of the society. The spirit which was in the society dancers seized the novice and he vanished to the accompaniment of whitles. Later, he was heard and seen about the village under the influence of the spirit and without voluntary control over his actions. The state which overcame him corresponded to the vision or hallucinatory experience of a solitary guardian spirit seeker. Society members then enticed the novice, through his spirit, to return to the house where they captured him. His behavior was sometimes so violent that he had to be forcibly restrained When his ecstacy or frenzy had been brought under control, he danced for his spirit power, and the songs associated with it were sung for him by the society members. Each initiate received an individual dance, song, name, and symbol from the tutelary. This parallels the individual and personal manifestation of power received by a solitary seeker (Viola Garfield, 1939 : 298). 
ges. Ces sociétés et prérogatives correspondent pour l'essentiel aux sociétés kwakiutl. Mais le simhalait présente un cas particulier qui remet en question la notion d'un centre unique de développement des sociétés secrètes, souligne l'importance des traditions locales et illustre le rôle rituel des chefs dans la société tsimshian. L'histoire du simhalait est complexe, obscure, et sa reconstruction reste problématique : le simhalait est souvent traité comme une société secrète, car il s'agit d'une initiation dont certaines des formes rappellent ce qui se passe chez les Mitla ; mais il ne s'agit ni d'une société séparée, ni de rites secrets. On ne le retrouve pas non plus sur la liste des sociétés kwakiutl. En fait, il ne s'agit pas vraiment d'une société secrète, mais d'un complexe particulier aux Tsimshian. D'après Franz Boas, le simhalait ne se confine pas aux cérémonies des sociétés secrètes mais "s'obtient quand une personne acquiert le premier esprit-gardien de son clan et célèbre la cérémonie qui appartient à cet événement». Par ailleurs, Boas nous apprend aussi que le nom de la société du simhalait est le seul qui ne soit pas d'origine kwakiutl et que le simhalait n'est pas mentionné dans les légendes relatant l'origine des sociétés secrètes (Boas, 1895 : 651-52). Phillip Drucker va plus loin que Boas puisqu'il décrit le simhalait, non pas comme une société secrète, mais «plutôt comme une célébration au cours de laquelle on expose les blasons du clan». Drucker modifie ensuite cette définition, inexacte en partie comme nous le verrons, en ajoutant:

L'enfant en honneur duquel on prépare cette exposition est inspiré par le chef, le blason est présenté par un danseur, puis le chef reprend l'esprit (avec lequel l'enfant a été inspiré). L'enfant porte durant quelques jours une couronne de cèdre rouge qu'il retire ensuite sans autres formalités. Ceux qui ont été inspirés de cette façon ne forment pas d'assemblée ou de groupe exclusif La célébration semble résulter d'un transfert plutôt évident d'un modèle déduit des sociétés secrètes et appliqué à une autre institution, la présentation des blasons du clan (Drucker, $1944: 222)$.

Viola Garfield divise le simhalait en deux parties distinctes, le ts'iik et le simhalait. Le terme ts'iik, qui correspond au terme bella bella tseka, est probablement emprunté aux Kwakiutl, mais désigne la cérémonie qui prend place lors de la première initiation des enfants; le terme simhalait désigne la seconde initiation, en tous points semblable à la première ; c'est pourquoi je pense que le terme ts'iik 
a simplement remplacé le terme simhalait plus ancien. Aujourd'hui, simhalait est le terme qui désigne une danse de bienvenue qui fait obligatoirement partie des \&tes modernes chez les Tshimshian. Mais il s'agit là seulement de l'un des éléments de la cérémonie traditionnelle du même nom. Traditionnellement en effet, le simhalait est divisé en trois parties : d'abord par une série de danses naxnoq, ensuite par une séance d'initiation, enfin par une danse simhalait ou danse de bienvenue. Ces trois rituels ne prennent pas nécessairement place dans l'ordre indiqué et, sous des formes un peu différentes, ils peuvent être retrouvés à d'autres occasions. Tous trois font appel au chef en tant que halait ou ritualiste, plutôt qu'en tant que simooget ou figure politique.

Les danses naxnoq sont exécutées par les chefs ou leurs délégués, souvent leurs héritiers. Elles dramatisent la visite d'un naxnoq (c'est-à-dire d'un esprit ou puissance), dont le chef (homme ou femme) porte le nom parce qu'il en a reçu le droit par héritage, parce qu'il a rencontré le naxnoq dans une quête rituelle, ou parce que le naxnoq s'est manifesté à lui de façon spontanée et lui a laissé un don ou pouvoir quelconque, ou simplement de la chance dans ses entreprises. Cette rencontre avec le naxnoq est officialisée dans un nom-naxnoq, un concept typiquement tsimshian qu'il ne faut pas confondre avec le blason comme le fait Phillip Drucker. En effet, les blasons ne sont pas des naxnoq : bien qu'ils en soient souvent des représentations, ils ne rendent pas la puissance des naxnoq effectivement présente, ne faisant que les rappeler ou les commémorer. Chaque nom-naxnoq est accompagné d'une danse ainsi que d'un chant qui figure une représentation précise du naxnoq ou la dramatisation d'une rencontre avec cet esprit ou entité nonhumaine - l'entité est représentée par le danseur masqué et costumé. Le don ou pouvoir reçu est représenté de façon aussi dramatique et théâtrale que possible ; il arrive même que cela soit truqué. Les danses-naxnoq ont été réintroduites dans les \&tes gitksan depuis une dizaine d'années. Cependant, elles sont très anciennes puisqu'on les trouve mentionnées dans les récits de voyage de l'explorateur espagnol Jacinto Caamano qui visita la région durant l'été de 1792. Caamano fut invité par le chef tsimshian Jammisit à assister à une fête durant laquelle lui et d'autres chefs, parmi ses invités d'honneur, présentèrent une série de sketches et de danses costumés et masqués, qui ressemblent de façon évidente aux danses et représentations décrites par Viola Garfield et Marius Barbeau. 
Un deuxième élément du simhalait est l'initiation des jeunes. L'initiation du simhalait a lieu seulement en hiver durant la saison sacrée. Le rituel dépend pour les détails du nom sacré du chef ou des chefs qui officient, mais le scénario reste le même : après dramatisation de son nom-naxnoq correspondant à son titre de wihalait faisant en général référence au domaine céleste, le chef jette ou lance sur les jeunes novices une partie de sa puissance. Les novices disparaissent plusieurs jours, puis réapparaissent capables de danser leur nouveau don ou même de présenter l'un des naxnoq de leurs lignages. La décision d'initier une personne est prise par ses parents et ses chefs. L'initiation, publique, est suivie ensuite par un enseignement formel de plusieurs jours ou plusieurs semaines.

Bien qu'on soit en pleine société matrilinéaire, le chef qui officie n'est pas nécessairement l'oncle maternel des enfants, mais le chef du village (ou même un chef d'un village voisin). Son affiliation clanique ne joue aucun rôle, un aspect important qui confirme l'idée, développée par Marjorie Halpin 16, qu'au début du XIXe siècle certains éléments de la chefferie tsimshian étaient orientés au-delà du lignage, et que le pouvoir politico-religieux de certains chefs avait largement dépassé le groupe de parenté, ce qu'Halpin démontre par l'étude des blasons et l'ambiguïté de certaines images qui marquent alors, selon elle, le rang plutôt que l'affiliation au groupe de parenté. L'initiation elle-même, que Drucker appelle inspiration, est composée de plusieurs éléments : certains de ces éléments tels la disparition des initiés, le concept de possession et l'usage des flûtes et sifflets rappellent les rituels des sociétés secrètes ; d'autres, comme la nature des entités qui possèdent temporairement le novice, sont indubitablement liés à la quête d'un espritgardien (encore pratiquée, pour la période décrite par Viola Garfield et Franz Boas, par les chamanes et par les chasseurs et tous les individus désireux d'accroître leur chance, leur succès, leur richesse, ou leur rang) ; d'autres enfin semblent être des techniques rituelles empruntées à d'autres cérémonies, en particulier la façon, dont le naxnoq ou l'esprit est littéralement jeté sur le novice, rappelle des rituels de bienvenue durant lesquels les chefs ou les ritualistes se lancent réciproquement leurs pouvoirs magiques ou surnaturels, les font apparaître et disparaître

16 Marjorie Halpin, dans sa thèse de doctorat, a apporté des précisions sur l'existence de ces chefs et la façon dont le système de blason s'est partiellement adapté à l'expression du rang plutôt qu'à celle de l'appartenance au lignage. 
au grand étonnement de la foule qui n'est pas censée être au courant des trucs de prestidigitation employés par les acteurs.

Le troisième élément du simhalait est une danse durant laquelle le chef souhaite bienvenue et paix à ses invités en secouant sur eux, à l'aide de mouvements de tête saccadés, le duvet d'aigle placé dans le panier qui couronne sa coiffure cérémonielle. Cette danse ainsi que le costume, qui l'accompagne de façon obligatoire, sont identiques à la danse et au costume mentionnés par Boas pour certaines danses kwakiutl et par McIlwraith pour certaines danses bella coola. On retrouve aussi le même costume chez les Haida et les Tlingit. Le costume amhalait du danseur est composé d'objets précis ; citons, en particulier, le hochet de chef en bois sculpté de façon extrêmement sophistiquée, qui représente un corbeau sur le dos duquel un humain allongé et un animal ou naxnoq sont reliés par leur langue commune, une cape « chilkat » en laine de chèvre sauvage tissée et teinte, et la coiffure cérémonielle amhalait faite d'un fronteau de bois sculpté et surmontée d'une couronne-panier en moustache d'otarie et attachée à une traîne de peaux d'hermine, complètent l'essentiel du costume. Ces objets sont toujours portés ensemble, que ce soit dans les descriptions ethnographiques ou dans les photographies, et ils ne sont jamais associés, chez les Tsimshian, à des masques. On sait que les capes « chilkat » étaient tissées à l'origine, en dépit de leur nom tlingit, par les Tsimshian qui les revendaient ensuite à leurs voisins. Les capes ainsi que les coiffures cérémonielles n'étaient pas décorées d'un motif blasonné, bien qu'elles puissent être utilisées comme telles, et pouvaient donc être transmises à des personnes appartenant à des lignages ou même des phratries différentes de celle du propriétaire originel. Des mythes tlingit mentionnent que les éléments du simhalait ont été découverts par les Tsimshian. Le hochet fut, quant à lui, inventé, d'après les informateurs de Marius Barbeau, par une famille de la rivière Nass. D'après Barbeau ${ }^{17}$, un Niska eut la vision d'un monstre émergeant d'un lac, vi-

17 "Adyaigwerh introduced dancing with the narhnorh (spirit), where these rattles were used. He was a Kanhade (Raven) of the Gitrhateen (tribe). When he gave a narhnorh to the chiefs he gave them a rattle, and it is then that the rattles were used, not otherwise. The Larhkibu (Wolves) of Gitrhateen tried to make a narhnorh themselves, without the permission of the Kanhade, and (one of them in consequence) was killed. This was Adinaw, who was the first one (of them) to try. Adiyaigwerh introduced this (kind of) dancing long before the arrival of the white man. Old Mathew Nass (was among those) who 
sion qu'il représenta sous forme du premier « hochet de chef » qui fut ensuite vendu en de nombreux exemplaires aux chefs tsimshian. L'événement se situerait au milieu du XVIIIe siècle ${ }^{18}$. Ajoutons que le hochet de chef le plus ancien dont nous ayons connaissance fut collectionné en 1804 par Baranoff chez les Tlingit.

Cette présentation très rapide de l'histoire des divers complexes cérémoniels indique que le simhalait est non seulement d'origine tsimshian, mais aussi qu'il précède, de plusieurs décennies au moins, l'introduction des sociétés secrètes. je pense que les danses naxnoq sont probablement encore plus anciennes. De nouvelles études montreront peut-être que le simhalait a pu voyager du nord au sud à l'inverse des sociétés secrètes puisqu'on retrouve des traces de rituels similaires chez les Kwakiutl. Ceux-ci affirment que ces rituels leur proviennent des peuples du Nord ${ }^{19}$. Ces études confirmeront probablement l'hypothèse de Marjorie Halpin selon laquelle le développement d'une chefferie tsimshian, plus inclusive que la chefferie des lignages, s'appuie sur l'évolution du rôle de ritualiste pour les chefs. Ces données nous permettent, en tout cas, d'entrevoir plus concrètement la complexité du contexte dans lequel opèrent les chamanes de la côte Nord-Ouest, ainsi que quelques-uns des facteurs qui nous permettraient de les définir de façon plus précise, en particulier leur position vis-à-vis de la vie cérémonielle de leur communauté.

saw the white people come. His brother was Hlederh, who saw the whiteman arrive, at the time when the Hudson's Bay Company was established at Graveyard Point (in 1831) (Barbeau, Manuscript on Raven rattle Tsimshian accession files).

18 Marius Barbeau a récolté pour le Musée national de l'Homme à Ottawa toute une série de masques naxnoq comprenant des descriptions des danses, des trucs de prestidigitation et des chants qui accompagnaient ces masques. À partir de ces informations Marjorie Halpin reconstitua les différences fondamentales entre le blason et le nom-naxnoq, et décrit les danses-naxnoq comme des charades. Un aspect important de ces " charades » est l'humour, voire la blague qui préside à leur présentation, sans pour autant nuire au rituel. Il s'agit pour les chefs de démontrer à la fois ses droits héréditaires, ses aptitudes à manipuler des puissances extra-humaines, et la proximité de ces puissances et du monde naxnoq durant certaines périodes cérémonielles.

19 Le simhalait, d'après mes recherches chez les Tsimshian, aurait pu influencer les rituels kwakiutl correspondants, en particulier ceux du laolaxa. 
J'ai ailleurs décrit le chamane tsimshian plus en détail ${ }^{20}$, et comparé ses méthodes à celles des initiateurs des sociétés secrètes et des participants aux autres cérémonies. Cette comparaison 21 confirme la position sociale du chamane tsimshian et l'individualité de ses méthodes. Ce n'est pas parce que les chefs s'attribuent des pouvoirs similaires à ceux des chamanes que les chamanes sont considérés comme des chefs. Les chamanes eux-mêmes, c'est-à-dire les guérisseurs, conservent toujours et partout leur indépendance vis-à-vis des systèmes socio-politiques ou même religieux de leurs communautés. Peut-être représentent-ils la possibilité pour l'être humain de se dégager du système culturel, alors que les chefs et initiés représentent au contraire l'enculturation du surnaturel 22 ?

\section{RÉFÉRENCES}

$\underline{\text { Retour à la table des matières }}$

BARBEAU, Marius

1958 Medicine Men of the North Pacific Coast, Ottawa, National Museum of Canada, Bulletin No. 152, Anthropological Series No. 42.

n.d. Tsimshian Accession Files, Unpublished manuscript, Canadian Ethnology Service, National Museum of Manitoba, National Museums of Canada (1911-1927).

20 Pour une analyse plus précise des notions tsimshian de halait et naxnoq ainsi qu'une étude des méthodes chamaniques tsimshian, voir mon article sur le chamanisme tsimshian, "Tsimshian Shamanic Images ».

21 Mary Douglas, dans Purity and Danger, et Victor Turner, entre autres dans son article sur les rites de passage, ont posé les jalons d'une étude de la liminalité culturelle. Mais je m'inspire d'Edward Sapir pour oser poser la relativité de tout système culturel véritable et la possibilité - Sapir dit la nécessité pour l'être humain cultivé de dépasser (Sapir dit « to grow out of ») le contexte culturel. je pense que l'initiation à laquelle se soumet le chamane met en cause des processus d'enculturation et d'acculturation encore peu connus. Voir l'article d'Edward Sapir : « Culture Genuine and Spurious », 1932.

22 Guédon, "Tsimshian Shamanic Images », dans : 'Me Tsimshian Images of the Past, Views of the Present, édité par Margaret Seguin - publié prochainement par U.B.C. Press, Vancouver. 
1961 Tsimshian Myths, Ottawa, National Museums of Canada, Bulletin No. 174, Anthropological series No. 51.

BENEDICT, Ruth

1923 "The Concept of the Guardian Spirit in North America," American Anthropologist, 29.

BOAS, Franz

1897 The Social Organization and Secret Societies of the Kwakiutl Indians, Report of the United States National Museum for 1885, Washington, D.C., Smithsonian Institution.

1902 Tsimshian Texts, Washington, D.C., Bureau of American Ethnology, Bulletin No. 27 : 1-244.

1916 Tsimshian Mythology, based on texts recorded by Henri Tate, Thirtyfirst Annual Report of the Bureau of American Ethnology, 1909-1910, Washington, D.C., Smithsonian Institution.

1930 Religion of the Kwakiutl Indians, Part 2, Columbia University, Contributions to Anthropology, X, Ithaca, N.Y., Columbia University Press.

BURRIDGE, Kennelm

1979 Someone, No One : An Essay on Individuality, Princeton, Princeton University Press.

CURTIS, Edward S.

1915 The North American Indian, XI, Cambridge, The University Press.

DE LAGUNA, Frederica

1972 Under Mount Saint Elias : The History and Culture of the Yakutat Tlingit, Washington, D.C., Smithsonian Contributions to Anthropology, II. 
DRUCKER, Philip

1940 Kwakiutl Dancing Societies, University of California Anthropological Records, (6), Berkeley, California, University of California Press : 201230.

DUFF, Wilson, (ed.)

1959 Histories, Territories, and Laws of the Kitwancool, Anthropology in British Columbia, Memoir No. 4, Victoria, B.C. Provincial Museum.

ELIADE, Marcea

1936 Le chamanisme et les techniques archaïques de l'extase, Paris.

1961 "Recent Works on Shamanism : A review article”, History of Religions, I (summer 1981) : 152-86.

FURST, Peter

1978 Hallucinogens and Culture, San Francisco, Chandler and Sharp Publishers, Inc.

GARFIELD, Viola

1939 Tsimshian Clan and Society, University of Washington Publications in Anthropology, Seattle, University of Washington : 167-340.

1955 “Making a Bird or Chief's Rattle”, Davidson Journal of Anthropology, 1 (2) : 155-164.

1966 “The Tsimshian and their Neighbors”, Dr. Viola E. Garfield and Paul S. Wingert (eds.), The Tsimshian Indians and their Art, Seattle, University of Washington Press. 
GOLDMAN, Irving

1975 The Mouth of Heaven, an Introduction to Kwakiutle Religious Thought, Toronto, A Wiley-Interscience Publication, John Wiley and Sons.

GOFF, Stanislav

1976 Realms of the Human Unconscious, Observations from L.S.D. Research, New York, E.P. Dutton.

GUÉDON, Marie-Françoise

1974 “Chamanisme Tsimshian et Athapaskan : un essai sur la définition des méthodes chamaniques”, Proceedings of the First Congress of the Canadian Ethnology Society, J. Barkow (ed.), Mercury Series, 17, Ottawa, Canadian Ethnology Service : 181-221.

HALIFAX, Joan

1979 Shamanic Voices, a Survey of Visionary Narratives, New York, E.P. Dutton Publisher.

HALPIN, Marjorie

1973 The Tsimshian Crest System. A study Based on Museum Specimens and the Marius Barbeau and William Beynon Field Notes, Ph.D. Dissertation, Vancouver, University of British Columbia.

HARNER, Michael

1980 The Way of the Shaman, a Guide to Power and Healing. San Francisco, Harper and Row publishers. 
JENNESS, Diamond

1943 The Carrier Indians of the Bulkley River : Their Social and Religious Life, Bureau of American Ethnology, Bulletin No. 133, Washington, D.C., Smithsonian Institution.

JILEK, Wolfgang

1982 Shamanic Ceremonialism in the Pacific Northwest Today, Hancock House Publishers Ltd.

JONAITIS, Aldona

1981 "Creations of Mystics and Philosophers : the White Man's Perceptions of Northwest Coast Indian Art from the 1930s to the present”, Philosifica, $18: 13-39$.

LARSEN, Stephen

1976 The Shaman's Doorway, New York.

LÉVI-STRAUSS, Claude

1975 La voie des masques, Genève, Éditions d'Art Albert Skira.

MacDONALD, George

1982 Cosmic Equations on Northwest Coast Indian Art, Wilson Duff memorial volume, Victoria, British Columbia Provincial Museum.

McILWRAITH, Thomas

1948 The Bella Coola Indians, 2 vols, Toronto, University of Toronto Press. 
SAPIR, Edwards

1924 Culture, Genuine and Spurious, reprinted in D. Handelbaum (ed.), Selected Writings of Edward Sapir in Language Culture and Personality (1949), Berkeley et Los Angeles, University of California Press.

SWANTON, John

1950a Contributions to the Ethnology of the Haida, New York, Memoirs of the American Museum of Natural History, VIII (1).

1905b Haida Texts and Myth, Skidegate Dialect, Bureau of American Ethnology, Bulletin No. 29, Washington, D.C., Smithsonian Institution.

1908a Social Conditions, Beliefs, and Linguistic Relations of the Tlingit Indians, Twenty-sixth Annual Report of the Bureau of American Ethnology for the years 1904-1905, Washington, D.C., Smithsonian Institution.

1909 Tlingit Myths and Texts, Bureau of American Ethnology, Bulletin No. 39, Washington, D.C., Smithsonian Institution.

TURNER, Victor W.

1964 Betwixt and Between : the Liminal Period in Rites de Passage, The Proceedings of the American Ethnological Society, Symposium on New Approaches to the Study of Religion, University of Washington Press : 4-20.

1969 The Ritual Process, Bungay, Suffolk, England, Penguin Book Ltd.

VANCOUVER, Captain G.

1798 A Voyage of Discovery to the North Pacific Ocean and Round the World, Performed in the years 1790-1795, II : 388-389.

\section{Fin du texte}

